\title{
NL Understanding with a Grammar of Constructions
}

\author{
Wlodek Zadrozny and Marcin Szummer and Stanislaw Jarecki \\ and David E. Johnson and Leora Morgenstern * \\ IBM IResearch J)ivision \\ 'T.J.Watson Research Lab \\ Yorktown Heights, NY 10598 USA
}

\begin{abstract}
We present an approach to natural language understanding based on a computable grammar of constructions. A construction consists of a set of features of form and a description of meaning in a context. $\Lambda$ granmar is a set of constructions. This kind of grammar is the key clement of MINCAL, an implemented natural language speech-enabled interface to an online calendar system. The architecture has two key aspects: (a) the use of constructions, integrating descriptions of form, meaning and context into one whole; and (b) the separation of domain knowledge (abont calendars) from application knowledge (about the particular on-line calendar).
\end{abstract}

\section{Introduction: an overview of the system}

We present an approach to natural language understanding based on a computable grammar of constructions. $A$ construction consists of a set of features of form and a description of meaning in a context. $\Lambda$ grammar is a set of constructions. This kind of grammar is the key element of MINCAI, an implemented natural language speech-enabled interface to an on-line calendar system.

The system consists of a NL granmar, a parser, an on-line calendar, a domain knowledge base (about dates, times and meotings), an application knowledge base (about the calendar), a spoech recognizer, a speech generator.

In this paper we describe two key aspects of tho system architecture: (a) the use of constructions, where instead of separating NI processing into the phases of syntax, semantics and pragmatics, we integrate descriptions of form, meaning and context into one wholo, and use a parser that takes into account all this information (see [10] for details); (b) the separation of the domain knowledge (abont calendars) and the application knowledge (about the particular on-line calendar).

\footnotetext{
${ }^{*}$ M. Szummer and S. Jarecki are also from MIT.
}

\section{The dialogs}

The system allows users to engage in dialogs like: Schedule a meeting with Bob! At what time and date? On August 30th. At what time? At 8 . Morning or afternoon? - In the evcring.

The parser recognizes Schedule a meeting with Bob as an instance of sent (imp), the imperative construction consisting of a verb and an $\mathrm{NP}$, hore $n p$ (cvent). The context is used to prevent another reading in which with Bob rnodifies schedule, as in Dance a tango with Bob!. That is, a contextual rule is used which says that for calendar applications, poople do not modify actions or places. Context also plays an important role in understanding answers, e.g. At 8 . This is understood as a time expression (and not place or rate or something else) only because of the context.

The parameters of a meeting can be given in many ways, e.g. synonyms or different constructions can bo used, users can include as many parameters in a sentence as they wish, and the parameters can be given in any order. As a result there are about 10,000 ways of scheduling meetings (with a given set of parameters).

\section{How are the dialogs understood}

With respect to parsing, grammars of constructions can be parsed like "standard" grammars, except that the set of features is richer. Given a string (representing a sentence, a fragment of a discourse or a paragraph), the parser assigns it a construction. From this viewpoint, the situation is similar to "regular" parsing, and the possible algorithms are similar. We have implemented a prototype chart parser for construction grammars, discussed further in Section 3.

But, clearly, having understood the sentence as a linguistic entity in isolation is not the ultimate goal. Here the message of an utterance must be understood in the context of an intended action. This is done in two steps. First, the system determines the intended 
action and its parameters, using domain knowledge (meetings +time+places). Second, once all the parameters have been extracted from the dialog, the system executes the action. To do this, the program uses application-specific knowledge to translate the action and its parameters into a form that can be executed by the application (Xdiary).

\section{Constructions as data struc- tures}

A construction is given by the matrix:

$$
\left[\begin{array}{l}
\mathbf{N}: \text { name_of_construction } \\
{\left[\begin{array}{lll}
\mathbf{C} & : & \text { context } \\
\mathbf{V} & : & \text { structure } \\
\mathbf{M} & : & \text { message }
\end{array}\right]}
\end{array}\right]
$$

The vehicle $\mathbf{V}$ consists of formulas describing presence (or perhaps absence) of certain taxemes, or features of form, within the structure of the construction. Such a structure is given by a list of subconstructions and the way they have been put together (in all our exarnples this is concatenation, but there are other possibilities, e.g. wrapping). The context, $\mathbf{C}$, consists of a set of semantic and pragmatic constraints limiting the application of the construction. It can be viewed as a set of preconditions that must be satisfied in order for a construction to be used in parsing. The message, $\mathbf{M}$, describes the meaning of the construction, via a set of syntactic, semantic and pragmatic constraints.

To make this concrote, let us consider a fow $\mathrm{ex}$ amples. We begin with a simple "command construction" consisting of an action verb followed by its argument.

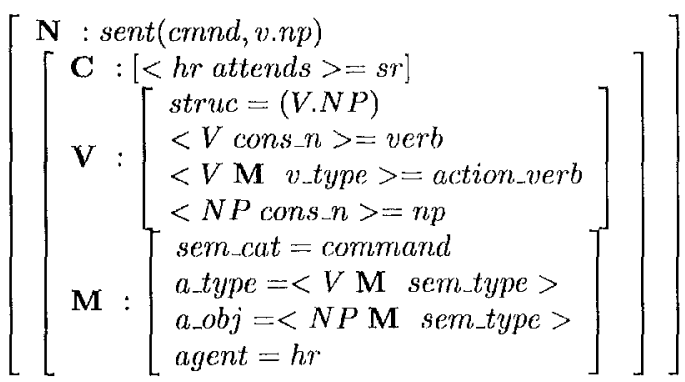

The context of the construction describes all situations in which the the hearer $h r$ (human or machine) is paying attention to the speaker $s r$ (a "ready" state). The feature struc is a list of variables and/or words/tokens; it is used to describe the structure of a construction, and its role is sirnilar to a rule in a generative grammar. (We will write names of variables in capital letters, e.g. $N P$, inside matrices of constructions). The attribute cons_n gives the name of a construction that could be assigned to a string. We use it here to say that the form of the construction can be described as a concatenation of two strings, of which one is a verb (construction) and the other an np (construction). Furthermore, the verb type $<V$ M vtype $>$ is "action_verb". (The expression $<V \mathbf{M}$ v type $>$ should be read "the $v$ type of the message of $V^{\prime \prime}$ ).

The message $M$ describes the meaning of the construction as that of a command in which the type of action is described by the meaning of the verb, and the object of the action is given by the meaning of the noun phrase. The attribute sem-type stands for the "semantic type" and we identify it currently with the word sense. Thus "erase the file" is understood as a command to delete the file, if $<$ erase $\mathbf{M}$ sem type $>=$ delete, but "erase the picture" might refer to the type of action associated with rub_out. In both cases the hearer $h r$ is supposed to be the agent of the action.

Constructions: from words to discourse

Words, phrases, and fragments of discourse can be analyzed as constructions. We view lariguages as colloctions of constructions which range from words to discourse. We claim that the same representation scherne can be used for all constructions.

The examples we are going to present have been developed with a specific purpose in mind, namely for scheduling calendar events. In other papers ([10] and [6]), wo have presented examples showing that we can give a good descriptions of non-standard constructions. However, in either case descriptions of mearings and contexts are general, and hence applicable to other tasks.

We now turn our attention to words. The verb "cancel" can be represented as follows:

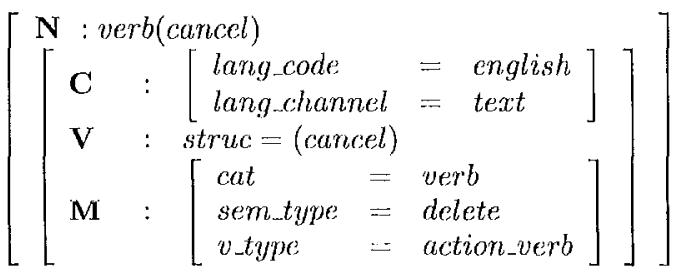

Notice that even simple words require context to be (properly) interpreted. In $\mathbf{C}$ we say that English text is expected (but in other cases it could also be Irench text, or lirench speech, etc.). Some aspects of context do not have to be oxplicitly specified and can be replaced by defaults.

Although the vehicle and the message are both very simple in this example, the simplicity of the message is a result of deliberate simplification. We have restricted it to the specification of the semantic type, identified with one sense of the word, and to describing the verb type of "cancel" as a verb of action. Notice that the other sense of "cancel" -. "offset, balance out" - would appear in another entry.

Of course, in reality, the lexical meaning of any word is a much more complicated matter [1]. For instance, in our lexicon the messages of words may contain many of the attributes that appetr in the 
explanatory combinatorial dictionary of Meleuk [7].

Discourse constructions: To illustrate discourse constructions, we consider the following dialog:

Have you arranged the room yet?

No, but I'll do it right away.

We view the pattern of the answer nobut.s as a discourse construction. It can represented by the following array of features:

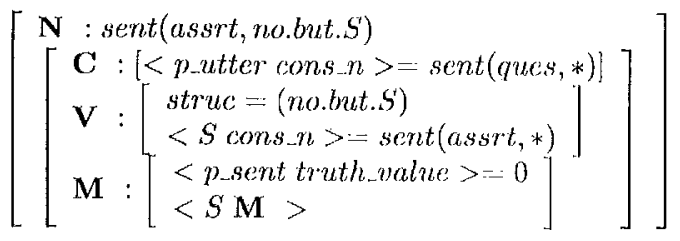

$\Lambda$ s we can see, the construction applies only in the context of a previously asked question, and its message says that the answer to the question is negative, after which it elaborates the answor with a senterce $S$.

\section{System Architecture}

\section{The parts}

MINCAL consists of a NI, grammar, a parser, a domain knowledge base (abont dates, times and meet ings), an on-line calendar (Xdiary), an application knowledge base (about Xdiary), a continuous spect recognizer (IBM, ICSS), a speech generator (Speech Plus, Text to Spech Converter), and the interfaces.

At present, the grammar consists of a fow humdred lexical constructions, and about 120 "productions", i.e. constructions describing combinations of other constructions. I It covers the basic forms of assertive sentences, but it emphasizes commands. Thus a command can, for example, be given either by v.np (also with "please", or "kindly"), or by an assertive sentence recognized as an indirect spech act ("I'd like to ...", "Ycora wants you to ...", etc.). 'The next large group of constructions covers Pl's, with particular emphasis on time and places. Finally, it covers a few discourse constructions, since it is important to deal with sentence fragments in dialogs, e.g. understanding "evening" as "in tho ovoning", when it is an answer to the question "when?".

\section{The interaction of the modules}

The calendar and the application knowledge base: Xdiary is an on-line calendar for which wo have not written a complete interface, but have focused on the three most important functions: appointment, moving, and cancoling appointments. Other functions, such as "to do" lists, window management, listing somobody's appointments, etc., can

\footnotetext{
${ }^{1}$ These are constructions we used in MINCAI. In addition in various experiments we have used a few dozen other constructions, e.g. those covering "open idioms" (see Section 4).
}

be dealt with in a similar fashion, and we plan to $\mathrm{ex}-$ tend the interface to deal with them. At this point the application knowledge base is very simple. It, consists of rules that say how to interpret the data given by the semantic interpreter, for instance the rules for formatting parameters and renaming slots (e.g. event duration - d duration). Such rules are nocessary, if the distinction between application and domain knowledge is to be maintained.

The domain knowledge base: This has two kinds of facts: (1) background ontology, i.e., is, basic facts about time and places, and (2) linguistic knowledge associated with the domain. The former includes such obvious facts as the number of days in a month, which month follows the other, that offices are places etc. 'The latter includes facts about how the language is used. For exarnple, the filters saying that places do not modify poople, so that $I$ want to mect my manager in the cafeteria can be unambiguously parsed, with "cafeteria" being a meeting place, and not an attribute of the manager.

The organization of knowledge: 'The issue of the organization of knowledge has been discussed at length in [8] and [9] and the formal model developed there is applicable in the present context. At this point, however, this formal model has only been implemented very crudely. Still the model is worth briefly discussing, because the conceptnal distinctions made guide our work and have important practical consequences. The most important thing about it is that we discard the model of background knowledge as a logical theory, and replace it by a model consisting of collection of theories and mochanisms for putting then together depending on circounstancess. 'T'hus, the usual, two-part logical structures, consisting of a metalevel and an object level, are augmented by a third level a referential level. The referential level is a partially ordered collection of thoories; it oncodes hackground knowledge in a way resembling a dictionary or an encyclopedia."

Parser, construction grammar and linguistic knowledge

Parser: Tho parser does not produce (syntactic) structural descriptions of sentences. Instead, it computes meaning representations. For example, it converts adjuncts directly into attributes of place, time, participant etc., once they can be computed, and thus the message of the sentence does not contain any information about how these attributes where expressed or about the attachment of PI's that appear in it. lor example, the sentence $I$ want you to arrange a conference in my office at 5 is analyzed as sent(assert, svoc), an assertive sentence consisting of a subjoct, a verb, an object and a complement.

\footnotetext{
'As usual, current situations are described on the object level, and the motalevel is a place for rules that can eliminate sorne of the models permitted by the object level and the reforential level.
} 
The latter and the message of the imperative that is passed to sent(assert, svoc) does not contain any structural information about the attachment of the PPs. This message is combined with the messages of the verb and the noun, yielding

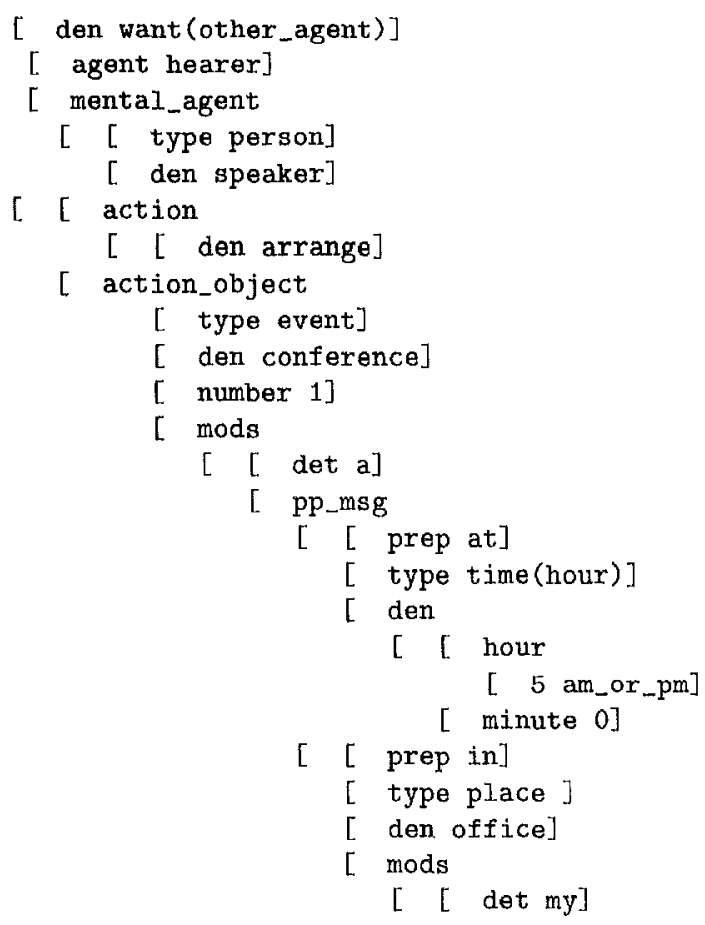

This result of parsing is then interpreted by the domain interpreter to produce:

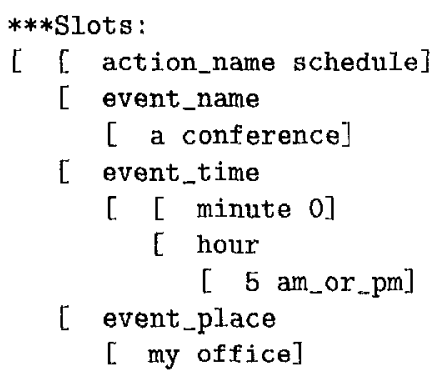

Application-specific defaults then produce yet another interpretation where, in addition to filling the slots of Xdiary, [ hour [5 am_or_pm] ] is interpreted as [ hour [17]].

The parser is a chart parser, working left to right, with no lookahead. The grammar is I-attributed, i.e., has has both synthesized and imherited attributes, but each irherited attribute depends only on inherited attributes of the parent or attributes of the sisters to the left. Hence, although the parser does not have a lookahead step at present, such a step can be added following [2].

\section{Comparisons with related work}

Linguistic arguments for constructions-based grammars has been worked out chiefly by Ch. Fillmore and his colleagues (cf. [3]). Their motivation for advocating such an approach comes from the fact that typical generative theories of grammar cannot deal properly with open idions illustrated by constructions such as:

The more carefully you work, the easier it will get.

Why not fix it yourself?

Much as I like Ronnie, I don't approve of anything he does.

It's time you brushed your teeth.

Him be a doctor?

The same is true about even so-callod robust parsers of English. The reason for this failure can be attributed to the fact that expressions like these "exhibit properties that are not fully predictable from independently known properties of its lexical makeup and its grammatical structure" - [3], p.511. However we do not need a list of "strange" constructions to conclude that thoroughly integrating syntax with semantics and pragmatics could provide us with a better handle on natural language understanding. On a closer examination "normal" constructions exhibit enough complexity to warrant the new approach (see [10] for details).

Jurafsky [4] has independently come up with a proposal for a computable grammar of constructions. We compare our work with his in [10]. Here, we limit ourselves to a fow remarks. What is common in both approaches is the contrality of the concept of grammatical construction as a data structure that represents lexical, semantic and syrutactic knowledge. However, there are important differences between the two formalisms. First, the actual data structures used to represent constructions are different. The most important difference has to do with the presence of the context field in our version of the construction grammar. This allows us to account for the importance of pragmatics in representing many constructions, and to deal with discourse constructions.

Secondly, while Jurafsky acknowledges the noed for abstract constructions (pp.43-51), his abstract constructions (weak constructions) are not first class citizens - they are defined only extensionally, by specifying the set of constructions they abstract over, and their abstract meaning (e.g. entity for NOUN). They are used to simplify descriptions of constituents of other constructions. However, because they do not have a separate vehicle part, they cannot be used to assign dofault mearings. For instance, since verb is defined as a collection of all verbs is + read + can$c e l+k n o w+l o o k-u p+\ldots$, it cannot be assigned a feature action_verb without introducing a contra- 
diction - its semantics is therefore given as RELA'IION / PROGESs. For us the important feature of "abstract" constructions is not that they simplify descriptions of other constructions, but that they have default meanings. ( $\Lambda$ similar critique of $[5]$ can be found in [10]).

\section{Summary of results}

Our approach to NIU is based both on linguistic arguments and on our dissatisfaction with the state of the art. State of the art systems typically are too "syntax-driven", failing to take context into account in determining the intended meaning of sertences. A related further weakness is that such systems are typically "sentence oriented", rather than "conversation/discourse oricnted". In our view, this makes even the rnost robust systems "brittle" and ultimately impractical.

'Io test whether a construction-based approach is feasible built a "complete" working system that would include a representation for constructions. To do this, we focused on the "calendar clomain", a domain with limited complexity and simple but not uninteresting semanties. We have chosen to deal with sirmple actions, and not o.g. with question answering, where deeper understanding would be necossary. ${ }^{3}$

\section{Our contributions:}

1. We have proposed a new kind of grammar conlputable construction grammars, which are neither semantic, nor syntactic. Instead, their "productions" combine lexical, syntactic, semantic and pragmatic information. ${ }^{4}$

2. Wo have described duta structures for constructions, and have shown that they can be effectively used by the parser. Note that the same data structure is used to concode the lexicon and the "syntactic" forms.

3. We have shown hou to parse with constructions. We have implemented a simple chart parsing algorithm, which can be easily extended to an Fearlylike parser, as long as the construction grarnmar remains L-attributed. We have found that even a simple parser of construction can be quite efficient. 'I'his is partly due to the fact that it does not require copying of all syntactic and semantic information from danghters to mothers; the goal of parsing consists in producing an interpretation, and structural information can be cliscarded once an interpretation of a phrase is produced. It is also worth emphasizing

\footnotetext{
${ }^{3}$ We have also thought about another possibility, that is, enhancing an IR system, e.g. with the understanding of date expressions.

${ }^{4}$ In what sense aro they "computable"? Although this adjective might suggest a formal molel with computational complexity results, etc., what we have in mind is pretty trivial: (1) the system actually computes the mossages of grammatical construction; (2) the grammars and constructions are well defined data structures, and parsing (combining all associated constructions in all possible ways) is decidable.
}

that invoking domain semantics drastically reduces the number of parses constructed.

4. We have proposed a modular architecture for $N L$ interfaces based on the division between linguistic knowledge, domain knowledge base, and application knowledge base. Based on our experience, we believe that this architecture should work in general for spcech-enabled interfaces for restricted domains.

\section{References}

[1] Computational Linguistics. Special Issue on the Lexicon, 1987. 13: 3-4.

[2] Nelson Correa. An extension of earley's algorithrn for s- and l- attributed grammars. In Proc. Intl. Conf On Current Issues in Computational Linguistics, pages 248-261. Penang, Malaysia, 1991.

[3] Charles J. Fillmore, Paul Kay, and Mary Catherine O'Connor. Regularity and idiomaticity in grammatical constructions. Langnage, 64(3):501-538, 1988.

[4] 1). Jurafsky. An On-line Computational Model of Sentence Interpretation. PhD thesis, University of California, Berkeley, 1992. Report No. UCB/CSD 92/676.

[5] Paul Kay. Even. Linguistics and Philosophy, 13(1):59-111, 1990

[6] M. McCord, Arendse Bernth, Shalom I appin, and Wlodek Zadrozny. Natural language procossing techrology based on slot grammar. In ternational Journal on Artificial Intelligence T'ools, L(2):229-297, 1992.

[7] I. Melcuk. Dependency Syntax: Theory and Practice. State University of New York Press, Albany, NY, 1988.

[8] Wlodek Zadrozny. Reasoning with background knowledge - a three-level theory. Computational Intelligence, 10(2), 1994. To appear.

[9] Wlodek Zadrozny and Karen Jensen. Semantics of paragraphs. Computational linguistics, 17(2):171-210, 1991.

[10] Wlodek Zadrozny and Alexis Manaster-Ramer. The significance of constructions. Subrnitted to Computational Iinquistics, 1994. 
